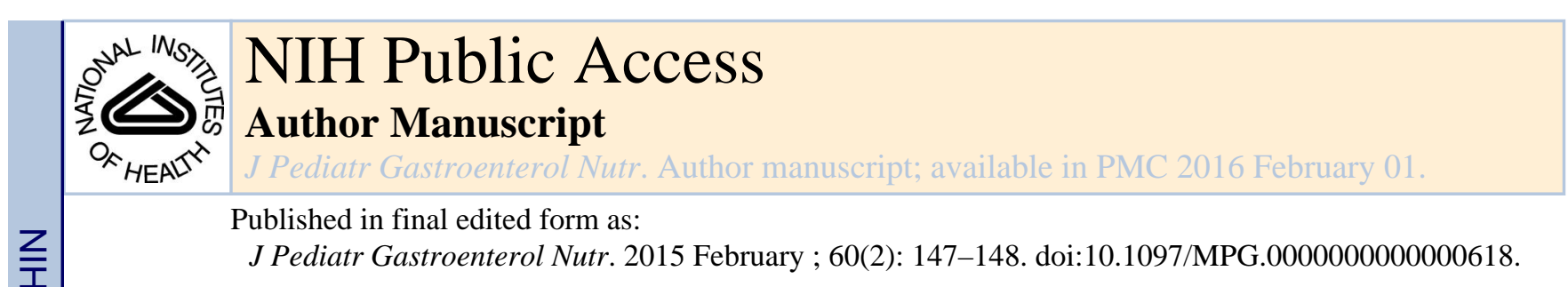

\title{
On the Origin of Pediatric Nonalcoholic Fatty Liver Disease
}

\author{
Patricia A. Ugalde-Nicalo, M.D., M.A.S. ${ }^{1}$ and Jeffrey B. Schwimmer, M.D. ${ }^{1,2,3}$ \\ ${ }^{1}$ Division of Gastroenterology, Hepatology, and Nutrition, Department of Pediatrics, University of \\ California, San Diego School of Medicine, San Diego, California \\ 2Department of Gastroenterology, Rady Children's Hospital San Diego, San Diego, California \\ ${ }^{3}$ Liver Imaging Group, Department of Radiology, University of California, San Diego School of \\ Medicine, San Diego, California
}

\section{Keywords}

nonalcoholic fatty liver disease; obesity; pregnancy; diabetes; nutrition; hepatic steatosis; neonates

At the time of diagnosis the typical patient seen by a pediatric gastroenterologist for nonalcoholic fatty liver disease (NAFLD) is 12 years-old. One of the most common questions that parents ask is, "when did this begin?" Rarely is there a clear answer, however, there is increasing evidence that NAFLD could begin at birth or even in utero. In this edition of the journal, Patel et. al. present a retrospective autopsy study evaluating the presence and degree of fetal hepatic steatosis and its association with maternal diabetes. ${ }^{1}$ They compared 33 stillborn babies delivered to women with diabetes (cases) to 48 stillborn babies of women matched for age without diabetes (controls). For the cases, maternal diabetes was a mixture of gestational diabetes as well as established type 1 and type 2 diabetes. The majority of these women were obese (61\%) compared to the control group (33\%). The main finding of this study was a substantially higher rate of steatosis among cases (79\%) versus controls (17\%). In addition, the severity of steatosis was greater in the cases and was positively correlated with fetal body weight independent of maternal obesity.

Two studies have evaluated hepatic steatosis in neonates through the use of magnetic resonance spectroscopy (MRS). Modi and colleagues reported that maternal body mass index (BMI) at conception was independently correlated with neonatal hepatic fat content. ${ }^{2}$ Brumbaugh et.al. reported on hepatic steatosis in 25 neonates born to women with obesity and gestational diabetes or born to women who were normal weight and did not have diabetes. Liver fat content was $68 \%$ higher in those neonates born to women with obesity and gestational diabetes. ${ }^{3}$ Surprisingly, neonatal adiposity was not correlated with neonatal liver fat in either study, suggesting that in fetal life the drivers for hepatic fat accumulation may differ from those for adipose storage. In animal models, maternal obesity and overnutrition show a strong association with the early-onset of NAFLD in offspring. In

Address correspondence to: Jeffrey B. Schwimmer, M.D., Division of Gastroenterology, Hepatology, and Nutrition, Department of Pediatrics, University of California, San Diego, 3020 Children's Way, MC 5030 San Diego, CA 92123, jschwimmer@ucsd.edu, phone: 858-966-8907, fax: 858-560-6798. 
nonhuman primates McCurdy et.al demonstrated that maternal obesity and a high fat diet during gestation promoted fetal hepatic steatosis and oxidative stress during the third trimester. ${ }^{4}$ Likewise in a mouse model, Bruce et.al. reported that exposure to a high fat diet in early development and post-weaning periods increased the risk for nonalcoholic steatohepatitis in adult offspring. Weaning these mice to a standard control diet failed to fully reverse NAFLD, suggesting a lasting impact of the maternal environment on pathways of hepatic lipid metabolism. ${ }^{5}$

Patel and colleagues' cohort included a majority of African-American women (54\%), followed by Caucasian (27\%) and a minor proportion of Hispanic (9\%). However, these findings are not consistent with the epidemiology of NAFLD known in children. Hispanic children have the highest rate of hepatic steatosis, followed by Caucasians, and then African American children who have the lowest rate. ${ }^{6}$ In addition, other observations have suggested that African American children and adolescents tend to show a lower degree of fatty liver disease, even when controlling for obesity and insulin resistance. ${ }^{7}$ Thus, whether these new data are generalizable or not is unclear. Additionally, if NAFLD begins at birth, we would expect to see greater numbers of infants, toddlers and young children with NAFLD. Based on data from SCALE, the prevalence of NAFLD is extremely low in this age group and in fact is actually quite low before 8 years of age. Fatty liver prevalence increases with age ranging from $0.7 \%$ for ages 2 to 4 years up to $17.3 \%$ for ages 15 to 19 years. ${ }^{6}$

Fetal liver development begins at week 4 of gestation. During most of gestation, the developing fetal liver is in constant flux. In the environment of maternal obesity, the fetoplacental unit develops under conditions of both excess nutrients and inflammation. The increased substrate load creates a concentration gradient driving lipid flux to the fetus. Excess fetal lipid exposure in early to mid-gestation, may therefore utilize the liver and other developing organs as ectopic sites of excess lipid deposition in the absence of adipose tissue, resulting in whole-body insulin resistance and susceptibility to fatty liver throughout life. The postnatal persistence of increased hepatic steatosis may result from changes to hepatic de novo lipogenesis (DNL), fatty acid oxidation or lipoprotein export. Maternal obesity appears to prime de novo lipogenesis. ${ }^{8}$ Bruce et.al. demonstrated reduced fatty acid oxidation in adult mice that had been exposed to a maternal high fat diet and those postweaning high fat diet were unable to clear increased levels of intrahepatic lipid. ${ }^{5}$ During this critical period when fetal development is "plastic", the fetus is constantly experiencing a rapid cell proliferation making it sensitive to environmental challenges. Therefore, neonatal overfeeding and post-weaning high fat diet could prime hepatic lipid synthesis pathways both developmentally and biochemically that are associated with the onset and long-term risk for NAFLD. ${ }^{8,9}$

So what can one say regarding when NAFLD begins? Clinically the mean age at presentation has decreased slightly from 13 to 12 years-old. ${ }^{10}$ Whether hepatic steatosis associated with the fetal environment persists to become clinical NAFLD or disappears throughout infancy is unknown. As mentioned, if the onset of NAFLD occurs in the prenatal or neonatal period, then the prevalence of NAFLD in younger children should be higher than reported estimates. However, NAFLD may remain undetected in this demographic, as it is a condition that can be relatively asymptomatic, and younger children are much less likely to 
have screening laboratories. If NAFLD does indeed begin at birth, it has broad implications for how we counsel women even before they are pregnant. Improvement in preconception nutrition as well as prenatal nutrition would be required. Moreover, infants that are born to women with diabetes- likely a high risk group- would warrant close monitoring of nutritional status throughout childhood with anticipatory guidance provided to enhance awareness of potential for NAFLD development.

\section{Acknowledgments}

Funding Source: The authors were supported by NIH grants including DK61734, DK088925, DK088831 and DK090350. The contents of this work are solely the responsibility of the authors and do not necessarily represent the official views of the National Institutes of Health

\section{Abbreviations}

NAFLD nonalcoholic fatty liver disease

\section{References}

1. Patel KR, White FV, Deutsch GH. Hepatic steatosis is prevalent in stillborns delivered to women with diabetes. J Pediatr Gastroenterol Nutr. 2014

2. Modi N, Murgasova D, Ruager-Martin R, et al. The influence of maternal body mass index on infant adiposity and hepatic lipid content. Pediatr Res. 2011; 702:87-90.

3. Brumbaugh DE, Tearse P, Cree-Green M, et al. Intrahepatic fat is increased in the neonatal offspring of obese women with gestational diabetes. J Pediatr. 2013; 162(2):930-6. [PubMed: 23260099]

4. McCurdy CE, Bishop JM, Williams SM, et al. Maternal high-fat diet triggers lipotoxicity in the fetal livers of nonhuman primates. J Clin Invest. 2009; 119:323-335. [PubMed: 19147984]

5. Bruce KD, Cagampang FR, Argenton M, et al. Maternal high-fat diet feeding primers steatohepatitis in adult mice offspring, involving mitochondrial dysfunction and altered lipogenesis gene expression. Hepatology. 2009; 50:1796-808. [PubMed: 19816994]

6. Schwimmer JB, Deutsch R, Kahen T, et al. Prevalence of fatty liver in children and adolescents. Pediatrics. 2006; 118:1388-1393. [PubMed: 17015527]

7. Santoro N, Feldstein AE, Enoksson E, et al. The association between hepatic fat content and liver injury in obese children and adolescents. Effects of ethnicity, insulin resistance and common gene variants. Diabetes Care. 2013; 36:1353-1360. [PubMed: 23275357]

8. Brumbaugh DE, Friedman JE. Developmental origins of nonalcoholic fatty liver disease. Pediatr Res. 2014; 75:140-147. [PubMed: 24192698]

9. Ji C, Dai Y, Jiang W, et al. Postnatal overfeeding promotes early onset and exaggeration of high-fat diet-induced nonalcoholic fatty liver disease through disordered hepatic lipid metabolism in rats. $\mathrm{J}$ Nutr Biochem. 2014

10. Molleston JP, Schwimmer JB, Yates KP, et al. Histological Abnormalities in Children with Nonalcoholic Fatty Liver Disease and Normal or Mildly Elevated Alanine Aminotransferase Levels. J Pediatr. 2014; 164:707-13. [PubMed: 24360992] 